\title{
Giant Epidermoid Cyst: A Rare Cause of Temporal Lobe Epilepsy
}

\author{
Vinicius Gomes Trindade ${ }^{1}$ Marcos de Queiroz Teles Gomes ${ }^{1} \quad$ Marcelo Prudente do Espirito Santo $^{1}$ \\ Manoel Jacobsen Teixeira ${ }^{1}$ Wellingson Silva Paiva ${ }^{1}$
}

${ }^{1}$ Division of Neurosurgery, University of Sao Paulo Medical School, Sao Paulo, Sao Paulo, Brazil

J Neurol Surg Rep 2017;78:e101-e105.

\begin{abstract}
Address for correspondence Vinicius Gomes Trindade, MD, Division of Neurosurgery, University of Sao Paulo Medical School, 201Cardeal Arcoverde street, Sao Paulo, SP 05407000, Brazil (e-mail: viniciustrindade@hotmail.com).
\end{abstract}

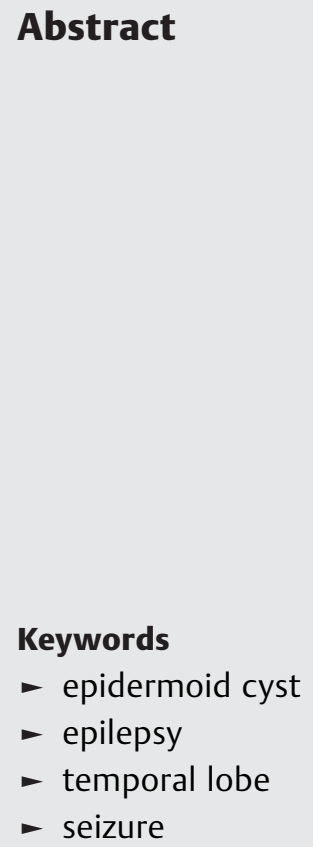

Introduction Epidermoid tumors represent approximately 0.3 to $1.8 \%$ of all intracranial brain tumors. Only $1.5 \%$ of all intracranial epidermoid cysts (ECs) invade the brain and secondary epilepsy is extremely rare. Since August 2014, a 59-year-old male smoker had been presenting bad smell feelings, totaling four episodes with sudden onset and duration of 2 minutes. On September 2014, after a sense of smell episode, it evolved into loss of contact and automatic movements followed by generalized tonicclonic movements. The brain magnetic resonance imaging revealed an extensive subtemporal lesion affecting anterior, middle, and posterior fossa with invasion of the choroidal fissure and projection to the temporal horn of the lateral ventricle. Pretemporal craniotomy with combined approaches, transsylvian and subtemporal, allowed for the excision of a white keratinized and softened lesion suggestive of EC. Discussion The optimal surgical strategy in individuals with ECs and seizures is not established. The evaluation of the cause and risk-benefit must be held to choose the appropriate surgical strategy: lesionectomy, lobectomy, or amygdalohippocampectomy. In this case, a lesionectomy was performed due to an absence of evidence of involvement of hippocampus and amygdala.

Conclusion Epilepsy secondary to ECs is a rare association. Lesionectomy can be an option with good results without increasing the morbidity.

\section{Introduction}

Epidermoid cysts (ECs) or tumors represent only 0.3 to $1.8 \%$ of all intracranial brain tumors. They are frequently located in the cerebellopontine angle (CPA, 40\%) and suprasellar cisterns $(18 \%)^{1}$ and less commonly in the middle cranial fossa, diploe, and spinal canal. They are formed during the third to fifth week of gestation due to faulty separation of the ectoderm and neuroectoderm, resulting in ectodermal inclusions during gastrulation. $^{2}$ ECs are usually benign and consist of a thin capsule of stratified keratinized squamous epithelium. ${ }^{3-5}$ Rare cases of squamous cell carcinoma arising from ECs have been reported. ${ }^{6}$ On magnetic resonance imaging (MRI), they usually appear as hypointense on T1-weighted imaging and hyper-

received

January 21, 2017

accepted

April 29, 2017
DOI https://doi.org/

10.1055/s-0037-1604281. ISSN 2193-6358. intense on T2- and diffusion-weighted imaging. The relative composition of cholesterol and keratin contributes to the MRI signal. ${ }^{7,8}$ Symptoms depend on their location and include hearing loss, dizziness, gait disturbance, trigeminal neuralgia, tinnitus, diplopia, visual impairment, apathy, headache, and gait ataxia. ${ }^{6,9,10}$ Rupture of an EC is a very unusual event, and can evolve into chemical meningitis and hydrocephalus. ${ }^{11}$ Epilepsy is an uncommon manifestation. We report a case of giant EC in which the unique symptom was epilepsy.

\section{Case Report}

A 59-year-old male smoker had been presenting bad smell feelings since August 2014, totaling four episodes with
License terms

Stuttgart · New York

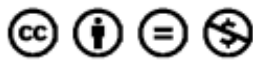




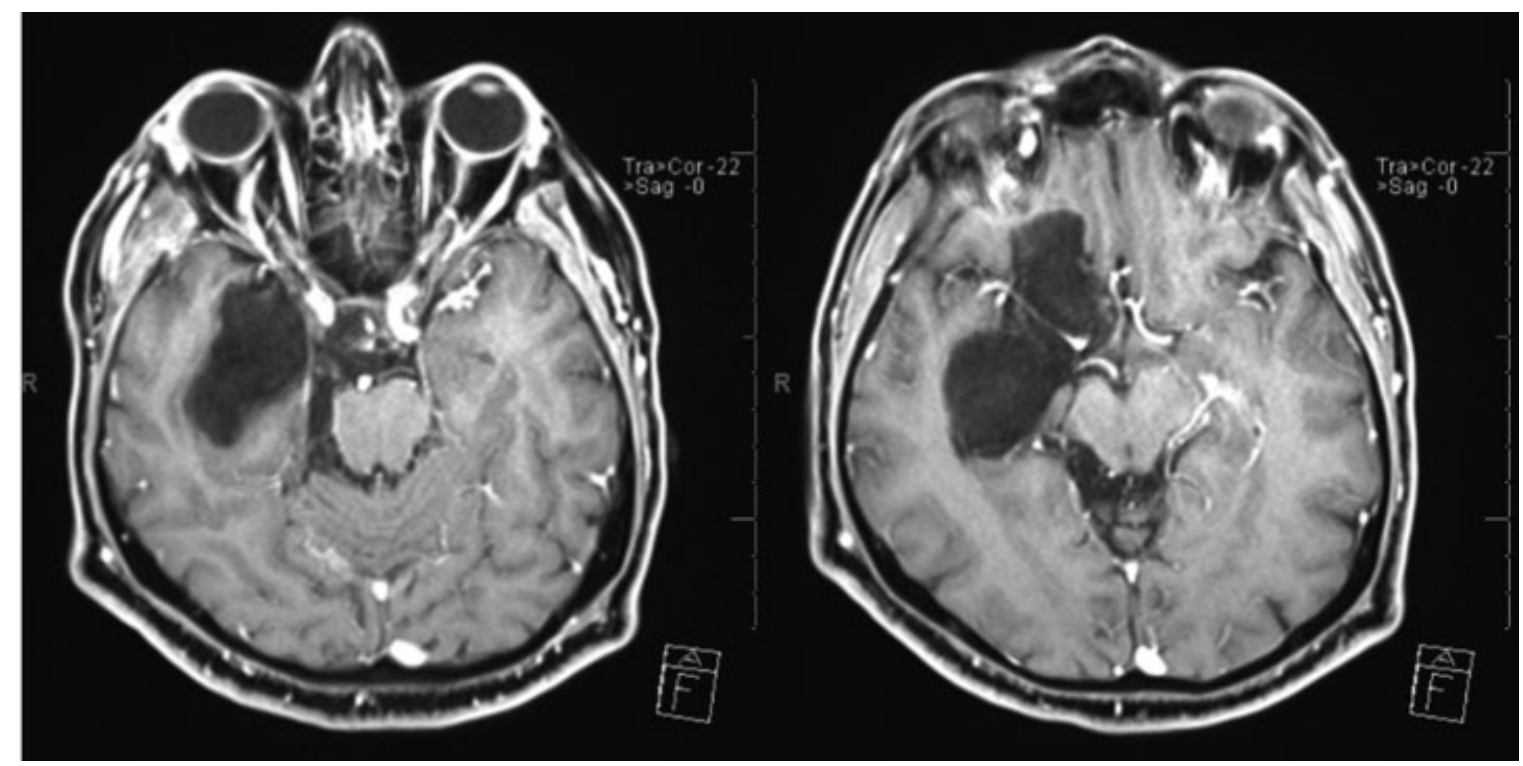

Fig. 1 Magnetic resonance imaging (MRI) with gadolinium shows a hypointense lesion with no contrast enhancement.

sudden onset and duration of minutes. In September 2014, after a sense of smell episode, it evolved into loss of contact and automatic movements followed by generalized tonicclonic movements. After this episode, he sought medical assistance. The neurological examination did not show any cognitive, strength, sensitivity, or coordination impairments. Evaluation of cranial nerves also showed no changes.

The brain MRI revealed an extensive subtemporal lesion affecting anterior, middle, and posterior fossa with invasion of the choroidal fissure and projection to the temporal horn of the lateral ventricle. Hyposignal on T1 sequence without contrast enhancement (-Fig. 1) and extremely high signal on T2
(-Fig. 2) were suggestive of EC or arachnoid cyst. Diffusion with marked restriction of water was very useful to diagnose EC (-Fig. 3).

The patient was then submitted to a pretemporal craniotomy with combined transsylvian and subtemporal approaches, allowing the excision of a white keratinized and softened lesion consistent with EC ( - Fig. 4). Since there was no evidence of brain tissue impairment (hippocampal and amygdala), we decided to remove only the lesion.

In the early postoperative period, the patient evolved into right abducens palsy without other neurological impairments and was discharged on the fifth postoperative day.

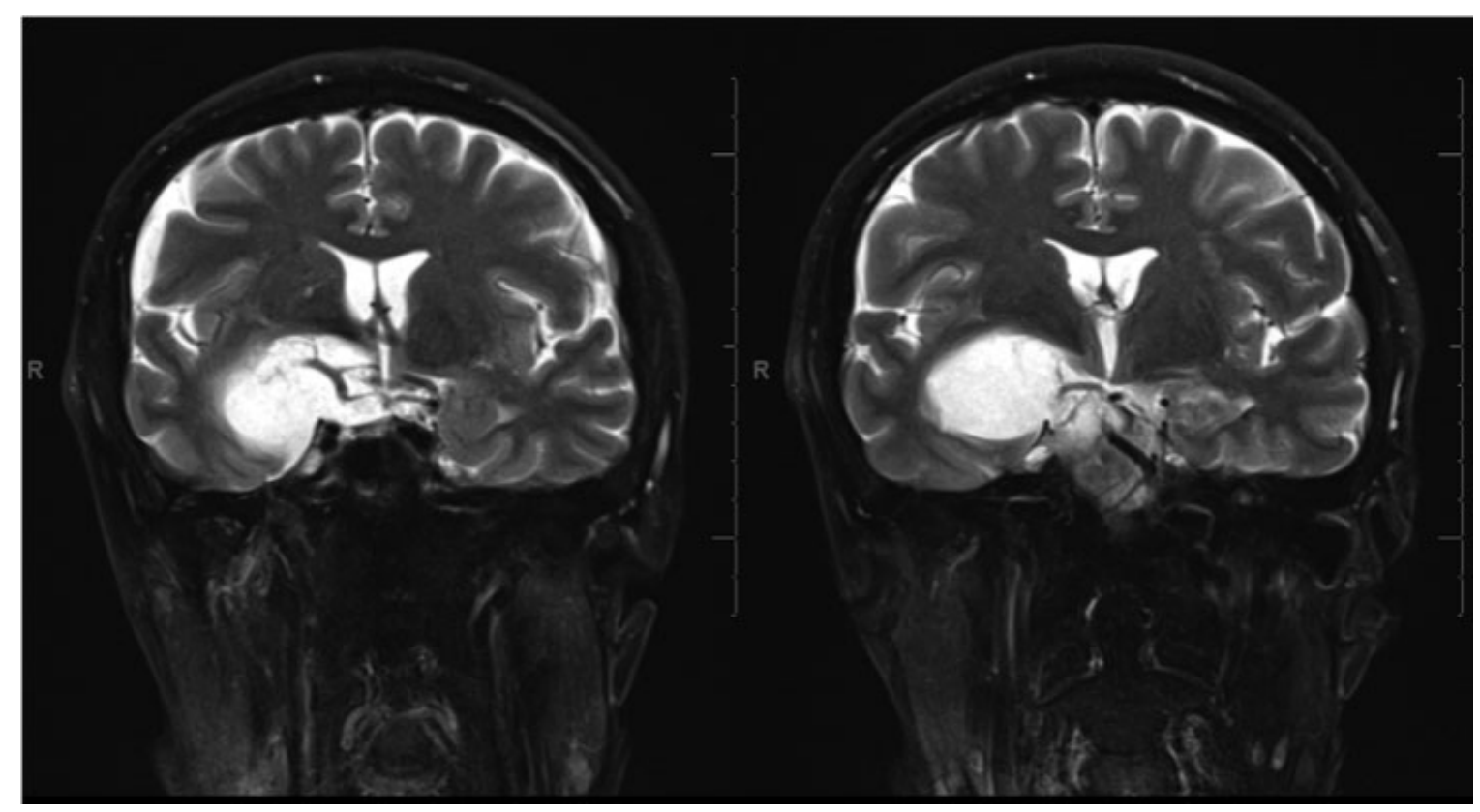

Fig. 2 Magnetic resonance imaging (MRI) coronal view. The lesion invades the temporal horn of the lateral ventricle through the choroidal fissure. 


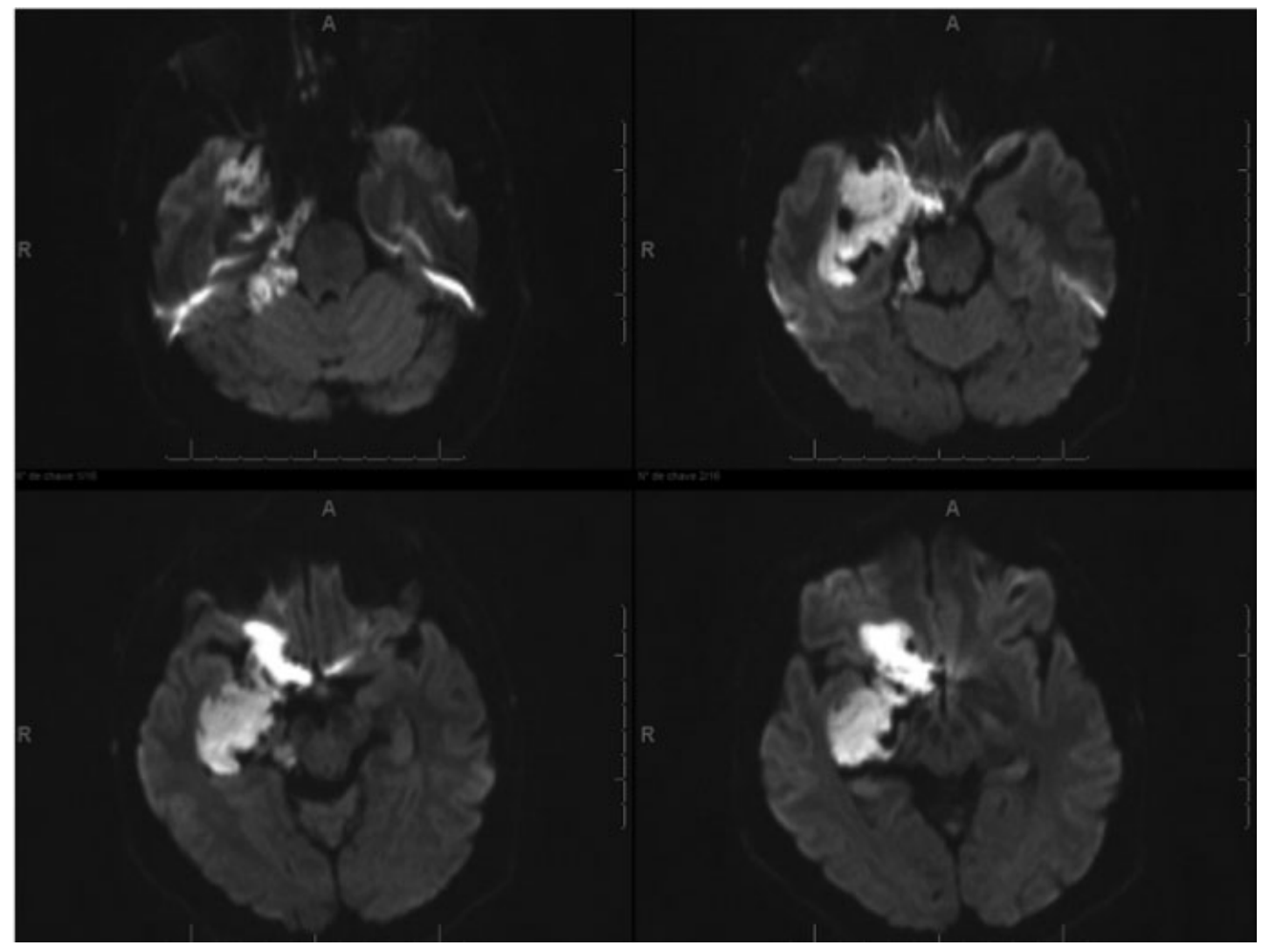

Fig. 3 Diffusion magnetic resonance imaging (MRI) shows restriction of water.

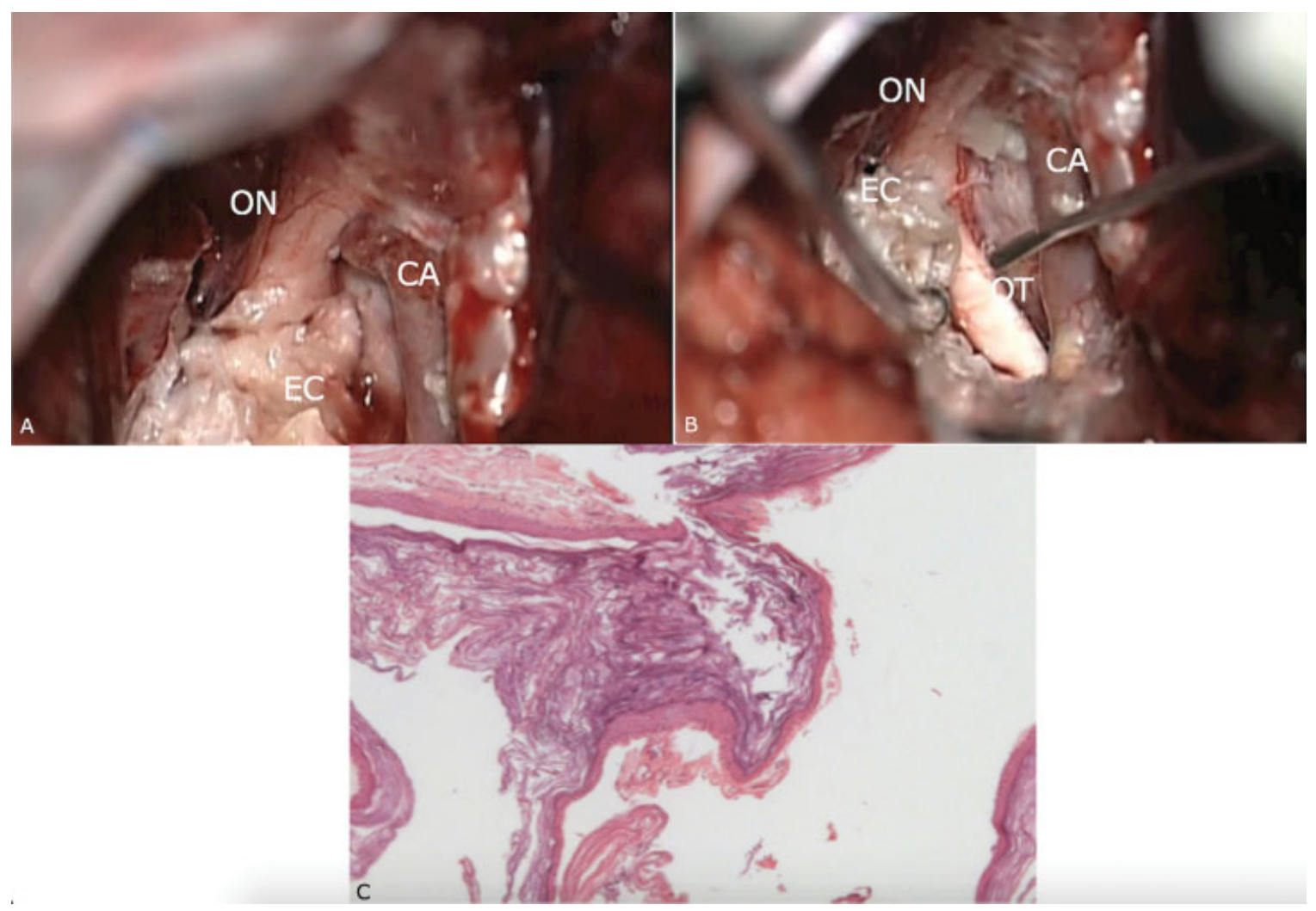

Fig. 4 (A and B) Surgical view shows the optic nerve (ON), optic tract (OT), carotid artery (CA), and the pearly epidermoid cyst (EC). (C) Microscopic view: hematoxylin and eosin (H \& S), magnification x50; stratified squamous epithelium and epidermal keratinous contents. 


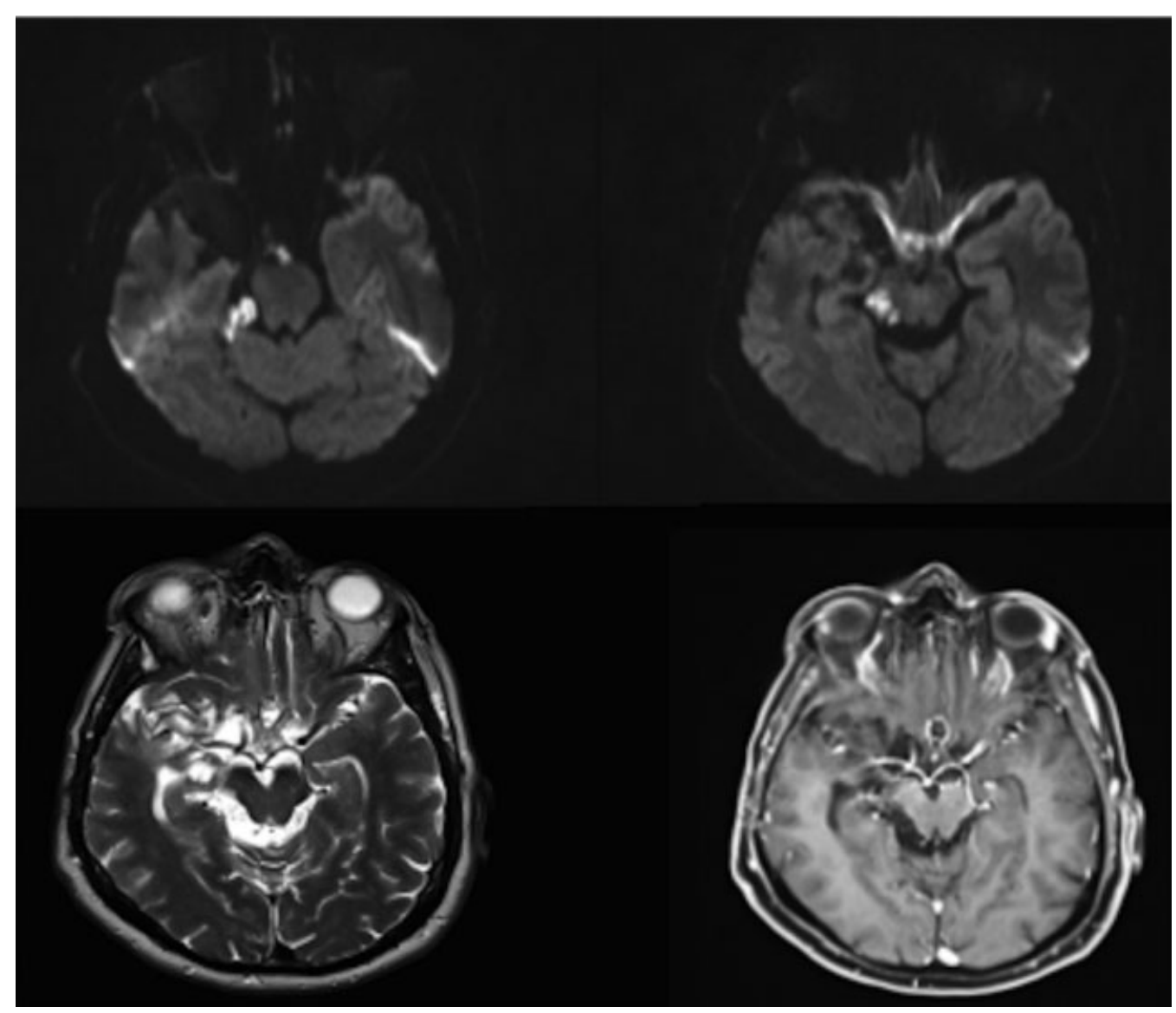

Fig. 5 Postoperative magnetic resonance imaging (MRI): small residual lesion in the cerebellopontine angle (CPA).

After 1 year, the patient exhibited no new seizures and improvement of abducens nerve palsy. The control MRI showed a small residual lesion in the posterior fossa (-Fig. 5).

\section{Discussion}

Epilepsy secondary to EC is rare and its epileptogenesis mechanism is not fully clarified. It may assign to infiltration of the brain tissue by the cyst, chemical meningitis, or an architectural change in epileptogenic areas.

Epidermoid tumors arising from or invading the parenchyma are exceedingly rare, ${ }^{12}$ accounting for less than $1.5 \%$ of all intracranial ECs, ${ }^{13}$ and may manifest with focal signs, epilepsy, and even a report of Holmes' tremor syndrome. ${ }^{14}$ While reviewing the literature, Hanft et al found 49 patients with exclusively intracerebral ECs. ${ }^{15}$ Tumors involved the temporal lobe in 16 patients, but only 4 of these patients developed seizures. ${ }^{15}$ Two cases of ECs infiltrating the temporal lobe with epileptic symptoms were successfully treated with lesionectomy alone. ${ }^{15}$ In 2012, a case of a patient with complex partial seizures caused by an EC and temporal lobe invasion through the choroidal fissure with hippocampal sclerosis was reported. In this case, the evidence of involvement of the hippocampus led to perform amygdalohippocampectomy associated with lesionectomy. ${ }^{16}$ Thus, the optimal surgical strategy in indivi- duals with ECs and seizures should be determined according to the tumor location and epileptogenic lesions.

The treatment of ECs involves radical surgical resection whenever possible, but keeping minimum residual lesion adherent in eloquent areas, cranial nerves, and small arteries to avoid irreversible neurological damage. The radiological follow-up with MRI is advisable and in cases of symptomatic regrowth, new resection is recommended. Radiotherapy is recommended in cases of malignization. ${ }^{17,18}$

The optimal surgical strategy in individuals with ECs and seizures is not established. The evaluation of the cause and riskbenefit must be held to choose the appropriate surgical strategy: lesionectomy, lobectomy, or amygdalohippocampectomy.

Our patient started with partial seizures and only one episode of generalization. The partial seizures were well controlled with the use of carbamazepine. MRI identified a bulky EC involving the posterior fossa, middle fossa with subtemporal extension, and invasion of temporal horn through the choroidal fissure and anterior fossa, but no sign of parenchymal involvement, such as edema or change in the signal on MRI. Based on the fact of no evidence of hippocampus or amygdala impairment in addition to the well-controlled crisis, we decided to perform a lesionectomy alone, leading to less surgical morbidity in the patient. A pretemporal craniotomy with transsylvian and subtemporal combined approaches was made, allowing for the subtotal 
excision of the lesion, with remnants of capsule attached to the brainstem and perfurant arteries. Patient evolved well with a transient abducens nerve paralysis, keeping crisis control after 1-year follow-up.

\section{Conclusion}

Epilepsy secondary to ECs is a rare association. The optimal surgical strategy in individuals with ECs and seizures is not established. Lesionectomy can be an option with good results without increasing morbidity in cases where parenchymal changes are seen on the MRI.

\section{References}

1 Tanriover N, Kacira T, Ulu MO, Gazioglu N, Oz B, Uzan M. Epidermoid tumour within the collateral sulcus: a rare location and atypical presentation. J Clin Neurosci 2008;15(08):950-954

2 Nair P, Sahu RN, Kumar R, Behari S, Nair AP, Srivastava AK. Large epidermoids of the quadrigeminal cistern: an experience of 15 consecutive cases and review of literature. Acta Neurochir (Wien) 2012;154(08):1391-1398

3 Feng R, Gu X, Hu J, et al. Surgical treatment and radiotherapy of epidermoid cyst with malignant transformation in cerebellopontine angle. Int J Clin Exp Med 2014;7(01):312-315

4 Hao S, Tang J, Wu Z, Zhang L, Zhang J, Wang Z. Natural malignant transformation of an intracranial epidermoid cyst. J Formos Med Assoc 2010;109(05):390-396

5 Lakhdar F, Hakkou M, Gana R, Maaqili RM, Bellakhdar F. Malignant transformation six months after removal of intracranial epidermoid cyst: a case report. Case Rep Neurol Med 2011;2011:525289

6 Nakao Y, Nonaka S, Yamamoto T, et al. Malignant transformation 20 years after partial removal of intracranial epidermoid cystcase report. Neurol Med Chir (Tokyo) 2010;50(03):236-239
7 Arai A, Sasayama T, Koyama J, Fujita A, Hosoda K, Kohmura E. Epidermoid cyst in Meckel's cave with unusual computed tomography and magnetic resonance imaging findings. Case report. Neurol Med Chir (Tokyo) 2010;50(08):701-704

8 Bohara M, Yonezawa H, Hanaya R, Takeshita S, Sumida M, Arita K. Posterior fossa epidermoid cysts presenting with unusual radiological appearances-two case reports. Neurol Med Chir (Tokyo) 2011;51(01):85-88

9 Aboud E, Abolfotoh M, Pravdenkova S, Gokoglu A, Gokden M, Al-Mefty O. Giant intracranial epidermoids: is total removal feasible? J Neurosurg 2015;122(04):743-756

10 Gopalakrishnan CV, Ansari KA, Nair S, Menon G. Long term outcome in surgically treated posterior fossa epidermoids. Clin Neurol Neurosurg 2014;117:93-99

11 Velamati R, Hageman JR, Bartlett A. Meningitis secondary to ruptured epidermoid cyst: case-based review. Pediatr Ann 2013;42(06):248-251

12 Mask-Bull L, Do B, Cattaneo JC. Brainstem epidermoid cyst in an individual with two previous intracranial epidermoid cysts: a rare diagnosis in a rare individual. Clin Neurol Neurosurg 2013; 115(09):1888-1890

13 Law EK, Lee RK, Ng AW, Siu DY, Ng HK. Atypical intracranial epidermoid cysts: rare anomalies with unique radiological features. Case Rep Radiol 2015;2015:528632

14 Menon B, Sasikala P, Agrawal A. Giant middle fossa epidermoid presenting as Holmes' tremor syndrome. J Mov Disord 2014; $7(01): 22-24$

15 Hanft SJ, Komotar RJ, Raper DM, Sisti MB, McKhann GM II. Epidermoid tumors of the temporal lobe as epileptogenic foci. J Clin Neurosci 2011;18(10):1396-1399

16 Hiraishi T, Oishi M, Kitaura H, et al. Epidermoid cyst involving the medial temporal lobe: surgical pathologic features of the epileptogenic lesion. Neuropathology 2012;32(02):196-201

17 Davies JM, Trinh VT, Sneed PK, McDermott MW. Radiotherapy for recurrent epidermoid cyst. J Neurooncol 2013;112(02):307-313

18 Nagasawa D, Yew A, Spasic M, Choy W, Gopen Q, Yang I. Survival outcomes for radiotherapy treatment of epidermoid tumors with malignant transformation. J Clin Neurosci 2012;19(01):21-26 